\title{
Discrete vs. continuum-scale simulation of radiative transfer in semitransparent two-phase media
}

\author{
Jörg Petrasch ${ }^{\mathrm{a}, *}$, Sophia Haussener ${ }^{\mathrm{b}}$, Wojciech Lipiński $^{\mathrm{c}}$ \\ a Department of Mechanical and Aerospace Engineering, University of Florida, Gainesville, FL 32611, USA \\ ${ }^{\mathrm{b}}$ Department of Mechanical and Process Engineering, ETH Zurich, 8092 Zurich, Switzerland \\ c Department of Mechanical Engineering, University of Minnesota, Minneapolis, MN 55455, USA
}

\section{A R T I C L E I N F O}

\section{Article history:}

Received 12 August 2010

Received in revised form

13 December 2010

Accepted 23 January 2011

Available online 1 February 2011

\section{Keywords:}

Radiative transfer

Volume averaging

Monte Carlo

Porous media

Continuum

Discrete

\begin{abstract}
A B S T R A C T
The mathematical formulation of the continuum approach to radiative transfer modeling in two-phase semi-transparent media is numerically validated by comparing radiative fluxes computed by (i) direct, discrete-scale and (ii) continuum-scale approaches. The analysis is based on geometrical optics. The discrete-scale approach uses the Monte Carlo ray-tracing applied directly to real 3D geometry measured by computed tomography. The continuum-scale approach is based on a set of continuumscale radiative transfer equations and associated radiative properties, and employs the Monte Carlo ray-tracing for computations of radiative fluxes and for computations of the radiative properties. The model two-phase media are reticulate porous ceramics and a particle packed bed, each composed of semitransparent solid and fluid phases. The results obtained by the two approaches are in good agreement within the limits of statistical uncertainty. The continuum-scale approach leads to a reduction in computational time by approximately one order of magnitude, and is therefore suited to treat radiative transfer problems in two-phase media in a wide range of engineering applications.
\end{abstract}

Published by Elsevier Ltd.

\section{Introduction}

Analysis of radiative transfer in complex two-phase media, in the limit of geometrical optics, is of fundamental importance to many engineering applications. Examples include high-temperature chemical reactions in packed beds [1-3], porous radiant burners [4-6], combustion furnaces for solid feedstock [7], high-flux solar receivers [8-10], and porous heat exchangers [11]. Traditionally, radiative transfer in such media has been modeled using the equation of radiative transfer with appropriate

\footnotetext{
Abbreviations: MC, Monte Carlo; ppi, pores per inch; RPC, reticulate porous ceramic; SAT, spatial averaging theorem; CT, computed tomography.

* Corresponding author. Tel.: +1 352392 9129; fax: +1 3523921071 .

E-mail address: petrasch@ufl.edu (J. Petrasch).
}

average radiative properties [12,13]. Average properties are determined either by directional and hemispherical flux measurements followed by inverse radiative transfer analysis or by direct numerical simulations based on the exact geometry of porous structures [14]. The latter approach was recently employed in conjunction with computed tomography by Zeghondy et al. [15], Petrasch et al. [16], and Haussener et al. [17-19]. Prior studies [14,17] pointed to the need for a precise definition of discrete-scale and continuum-scale properties and a rigorous derivation of continuum-scale equations of radiative transfer in multiphase semi-transparent media in the limit of geometrical optics. This has subsequently been carried out for twophase media in Ref. [20] and generalized to an arbitrary number of phases in Ref. [21]. In a prior study Consalvi et al. [22] carried out a similar averaging procedure limited to gray, diffuse, spherical particles. In contrast, the 


\begin{tabular}{|c|c|c|c|}
\hline \multicolumn{2}{|c|}{ Nomenclature } & $\psi$ & scalar quantity \\
\hline$A_{0}$ & specific surface, $\mathrm{m}^{-1}$ & $\Psi_{0}$ & threshold gray value for phase segmentation \\
\hline$d_{\mathrm{h}}$ & hydrodynamic diameter, $d_{\mathrm{h}}=4 \varepsilon_{\mathrm{p}} / A_{0}, \mathrm{~m}$ & $\Psi(\mathbf{r})$ & continuous gray level function \\
\hline I & $\begin{array}{l}\text { continuum-scale spectral radiative intensity, } \\
\mathrm{W} \mathrm{m} \mathrm{m}^{-3} \mathrm{sr}^{-1}\end{array}$ & \multicolumn{2}{|c|}{ Subscripts } \\
\hline \multirow[t]{2}{*}{$L$} & discrete-scale spectral radiative intensity, & & \\
\hline & $\mathrm{W} \mathrm{m} \mathrm{m}^{-3} \mathrm{sr}^{-1}$ & b & blackbody \\
\hline$\hat{\mathbf{n}}$ & outer unit normal vector & c & collision \\
\hline$N$ & number of rays & d & discrete \\
\hline $\mathbf{r}$ & discrete-scale position vector & in & incoming \\
\hline$\hat{\mathbf{s}}$ & unit directional vector & $i, j$ & phase index \\
\hline$V$ & averaging volume, $\mathrm{m}^{3}$ & int & interface \\
\hline $\mathbf{x}$ & continuum-scale position vector & $\mathrm{r}$ & reflection \\
\hline $\mathbf{y}$ & computed vector of normalized radiative flux, - & w & wall \\
\hline \multicolumn{2}{|c|}{ Greek symbols } & \multicolumn{2}{|c|}{ Superscript } \\
\hline$\epsilon$ & $\begin{array}{l}\text { normalized 2-norm of the normalized radia- } \\
\text { tive flux }\end{array}$ & $\stackrel{n}{\prime \prime}$ & $\begin{array}{l}\text { bi-directional } \\
\text { directional-hemispherical }\end{array}$ \\
\hline$\varepsilon_{\mathrm{p}}$ & porosity & + & forward \\
\hline$\theta$ & scattering angle & - & backward \\
\hline$\mu_{\mathrm{s}}$ & cosine of scattering angle $\theta$ & & \\
\hline$\tau$ & transmittance & & \\
\hline
\end{tabular}

procedure presented in Refs. [20,21] and applied in this paper is valid for an arbitrary discrete-scale geometry subject to geometrical optics at the discrete-scale.

In this paper, the continuum-scale modeling approach to radiative transfer based on volume averaging as proposed in Ref. [20] is for the first time applied to real geometries, and validated by discrete-scale numerical simulations using identical geometries. We apply the methodology formulated in Ref. [20] to determine average continuum-scale scattering coefficients and scattering phase functions of two model media: (1) silicon carbide reticulate porous ceramics (RPCs), and (2) a packed bed of calcium carbonate particles, both encountered in hightemperature solar thermochemical processing and other energy applications. Their exact discrete-scale geometries have been determined using computed tomography. The continuum-scale morphological and radiative properties, computed by a discrete-scale Monte Carlo (MC) raytracing method, are then used in continuum-scale MC simulations. Subsequently, the radiative fluxes obtained from the continuum-scale MC simulations are verified against those from discrete-scale MC simulations directly employing the exact discrete-scale geometry of the model media. A previous pertinent study on the numerical validation of a continuum-scale radiative transfer analysis of a suspension of large opaque spheres against a direct discrete-scale radiative transfer analysis, can be found in Ref. [23].

\section{Methodology}

The subsequent discussion is restricted to quasi-steady radiative transfer in semitransparent two-phase media in the limit of geometrical optics at the discrete-scale. Optical properties are assumed to be constant within each phase. Each phase is non-polarizing and the state of polarization can be neglected. Each phase is at local thermodynamic equilibrium and at rest as compared to the speed of light. Diffraction and dependent scattering effects are negligible. The medium is isotropic at the continuum-scale. The main governing relations derived in studies [20,21] are summarized below for the purpose of completeness of the present paper.

\subsection{Governing equations}

The above listed assumptions allow modeling discretescale radiative transfer in phase $i, i=1,2$, using the equation of radiative transfer (RTE) [12,13]:

$$
\begin{aligned}
\hat{\mathbf{s}} \cdot \nabla_{\mathbf{r}} L_{i}(\mathbf{r}, \hat{\mathbf{s}})= & -\left[\kappa_{\mathrm{d}, i}+\sigma_{\mathrm{s}, \mathrm{d}, i}\right] L_{i}(\mathbf{r}, \hat{\mathbf{s}})+n_{i}^{2} \kappa_{\mathrm{d}, i} L_{\mathrm{b}, i}(\mathbf{r}) \\
& +\frac{\sigma_{\mathrm{s}, \mathrm{d}, i}}{4 \pi} \int_{4 \pi} L_{i}\left(\mathbf{r}, \hat{\mathbf{s}}_{\mathrm{in}}\right) \Phi_{\mathrm{d}, i}\left(\hat{\mathbf{s}}_{\mathrm{in}}, \hat{\mathbf{s}}\right) \mathrm{d} \Omega_{\mathrm{in}}
\end{aligned}
$$

where $L_{\mathrm{b}, i}$ is the spectral blackbody intensity in vaccum; $\kappa_{\mathrm{d}, i}, \sigma_{\mathrm{s}, \mathrm{d}, i}$ and $\Phi_{\mathrm{d}, i}$ are the spectral absorption and scattering coefficients and the scattering phase function of phase $i$, respectively. It is assumed that the discrete-scale properties are known. The spectral subscript is omitted for brevity. The corresponding boundary condition at the interface between the phases $A_{\text {int }}$, where $\hat{\mathbf{s}} \cdot \hat{\mathbf{n}}_{i}<0$, is

$$
\begin{aligned}
L_{i}\left(\mathbf{r}_{\mathrm{int}}, \hat{\mathbf{s}}\right)= & \int_{\Omega_{\mathrm{in}} \hat{\mathbf{s}}_{\mathrm{in}} \cdot \hat{\mathbf{n}}_{i}>0} \rho_{i j}^{\prime \prime}\left(\hat{\mathbf{s}}_{\mathrm{in}}, \hat{\mathbf{s}}\right) L_{i}\left(\mathbf{r}_{\mathrm{int}}, \hat{\mathbf{s}}_{\mathrm{in}}\right) \hat{\mathbf{s}}_{\mathrm{in}} \cdot \hat{\mathbf{n}}_{i} \mathrm{~d} \Omega_{\mathrm{in}} \\
& -\int_{\Omega_{\mathrm{in}} \hat{\mathbf{s}}_{\mathrm{in}} \cdot \hat{\mathbf{n}}_{i}<0} \tau_{j i}^{\prime \prime}\left(\hat{\mathbf{s}}_{\mathrm{in}}, \hat{\mathbf{s}}\right) L_{j}\left(\mathbf{r}_{\mathrm{int}}, \hat{\mathbf{s}}_{\mathrm{in}}\right) \hat{\mathbf{s}}_{\mathrm{in}} \cdot \hat{\mathbf{n}}_{i} \mathrm{~d} \Omega_{\mathrm{in}}
\end{aligned}
$$


The spatial averaging theorem (SAT) is then applied to Eq. (1) $[24,25]$ :

$\frac{1}{V} \int_{V} \nabla_{\mathbf{r}} \psi_{i} \mathrm{~d} V=\nabla_{\mathbf{x}} \frac{1}{V} \int_{V} \nabla_{\mathbf{r}} \psi_{i} \mathrm{~d} V+\frac{1}{V} \int_{A_{\text {int }}} \psi_{i} \hat{\mathbf{n}}_{i} \mathrm{~d} A_{\text {int }}$

Using Eq. (2) in the second term on the RHS of Eq. (3) one obtains:

$$
\begin{aligned}
\hat{\mathbf{s}} \cdot & \nabla_{\mathbf{x}} I_{i}(\mathbf{x}, \hat{\mathbf{s}})=-\left[\kappa_{\mathrm{d}, i}+\sigma_{\mathrm{s}, \mathrm{d}, i}+\sigma_{\mathrm{s}, \text { int }, i i}+\sigma_{\mathrm{s}, \text { int }, i j}\right] I_{i}(\mathbf{x}, \hat{\mathbf{s}})+n_{i}^{2} \kappa_{\mathrm{d}, i} I_{\mathrm{b}, i}(\mathbf{x}) \\
& +\frac{1}{4 \pi} \int_{4 \pi} I_{i}\left(\mathbf{x}, \hat{\mathbf{s}}_{\mathrm{in}}\right)\left[\sigma_{\mathrm{s}, \mathrm{d}, i} \Phi_{\mathrm{d}, i}\left(\hat{\mathbf{s}}_{\mathrm{in}}, \hat{\mathbf{s}}\right)+\sigma_{\mathrm{s}, \text { int }, i i} \Phi_{\mathrm{int}, i i}\left(\hat{\mathbf{s}}_{\mathrm{in}}, \hat{\mathbf{s}}\right)\right] \mathrm{d} \Omega_{\mathrm{in}} \\
& +\frac{\sigma_{\mathrm{s}, \text { int }, j i}}{4 \pi} \int_{4 \pi} I_{j}\left(\mathbf{x}, \hat{\mathbf{s}}_{\mathrm{in}}\right) \Phi_{\mathrm{int}, j i}\left(\hat{\mathbf{s}}_{\mathrm{in}}, \hat{\mathbf{s}}\right) \mathrm{d} \Omega_{\mathrm{in}} .
\end{aligned}
$$

The following definitions of continuum-scale radiative properties are postulated [20,21] $(i, j=1,2$ and $i \neq j)$ :

$\sigma_{\mathrm{s}, \mathrm{int}, i i} \stackrel{\operatorname{def}}{=} \frac{\int_{A_{\text {int }} \hat{\mathbf{s}} \cdot \hat{\mathbf{n}}_{i}>0} \rho_{i}^{\prime} \bigcap(\hat{\mathbf{s}}) L_{i}\left(\mathbf{r}_{\text {int }}, \hat{\mathbf{s}}\right) \hat{\mathbf{s}} \cdot \hat{\mathbf{n}}_{i} \mathrm{~d} A_{\text {int }}}{I_{i}(\mathbf{X}, \hat{\mathbf{s}}) V}$

$\sigma_{\mathrm{s}, \mathrm{int}, i j} \stackrel{\operatorname{def}}{=} \frac{\int_{A_{\text {int }} \hat{\mathbf{s}} \cdot \hat{\mathbf{n}}_{i}>0} \tau_{i j}^{\prime} \bigcap(\hat{\mathbf{s}}) L_{i}\left(\mathbf{r}_{\text {int }}, \hat{\mathbf{s}}\right) \hat{\mathbf{s}} \cdot \hat{\mathbf{n}}_{i} \mathrm{~d} A_{\text {int }}}{I_{i}(\mathbf{X}, \hat{\mathbf{s}}) V}$

$\Phi_{\mathrm{int}, i i}\left(\hat{\mathbf{s}}_{\mathrm{in}}, \hat{\mathbf{s}}\right) \equiv \frac{\int_{A_{\mathrm{int}}: \hat{\mathbf{s}} \cdot \hat{\mathbf{n}}_{i}<0} \rho_{i j}^{\prime \prime}\left(\hat{\mathbf{s}}_{\mathrm{in}}, \hat{\mathbf{s}}\right) L_{i}\left(\mathbf{r}_{\mathrm{int}}, \hat{\mathbf{s}}_{\mathrm{in}}\right) \hat{\mathbf{s}}_{\mathrm{in}} \cdot \hat{\mathbf{n}}_{i} \hat{\mathbf{s}} \cdot \hat{\mathbf{n}}_{i} \mathrm{~d} A_{\mathrm{int}}}{(-4 \pi)^{-1} \sigma_{\mathrm{s}, \mathrm{int}, i i} I_{i}\left(\mathbf{x}, \hat{\mathbf{s}}_{\mathrm{in}}\right) V}, \quad \hat{\mathbf{s}}_{\mathrm{in}} \cdot \hat{\mathbf{n}}_{i}>0$

$\Phi_{\text {int }, j i}\left(\hat{\mathbf{s}}_{\text {in }}, \hat{\mathbf{s}}\right) \equiv \frac{\int_{A_{\text {int }} \hat{\mathbf{s}} \cdot \hat{\mathbf{n}}_{i}<0} \tau_{j i}^{\prime \prime}\left(\hat{\mathbf{s}}_{\mathrm{in}}, \hat{\mathbf{s}}\right) L_{j}\left(\mathbf{r}_{\text {int }}, \hat{\mathbf{s}}_{\mathrm{in}}\right) \hat{\mathbf{s}}_{\mathrm{in}} \cdot \hat{\mathbf{n}}_{i} \hat{\mathbf{s}} \cdot \hat{\mathbf{n}}_{i} \mathrm{~d} A_{\text {int }}}{(4 \pi)^{-1} \sigma_{\mathrm{s}, \mathrm{int}, j i} I_{j}\left(\mathbf{X}, \hat{\mathbf{s}}_{\mathrm{in}}\right) V} \quad \hat{\mathbf{s}}_{\mathrm{in}} \cdot \hat{\mathbf{n}}_{i}<0$.

In the limiting case of vanishing volume fraction of one of the two phases, the interfacial area $A_{\text {int }}$ and thus the interface-related radiative coefficients given by Eqs. (5) and (6) approach zero for this phase. Consequently, the system of two RTEs (4) reduces to a single RTE, written for the only remaining phase. In the limiting case of one opaque phase, the surface transmissivity becomes zero and Eq. (6) predicts zero interfacial scattering associated with refraction at the interface-it is replaced in the model by interfacial absorption and emission as discussed in Refs. [20,21].

\subsection{Determination of continuum-scale properties}

In the subsequent discussion, it is assumed that Eqs. (5)-(8) are independent of the local intensities $L_{i}$ and $L_{j}$ as long as $L_{i}$ and $L_{j}$ satisfy Eqs. (1) and (2). In this case, the average radiative properties would depend on the discrete-scale geometry and discrete-scale radiative properties only. Consider the averaging volume $V=A s$ consisting of a plane-parallel and thin slab of thickness $S$ that contains all relevant morphological features of the medium, i.e., its volume is at least equal to a representative elementary volume (REV) [26], while the slab is thin enough such that multiple reflections may be neglected. In the study [16], the REV of the RPC foam employed in this study was determined as $43 \times d_{\text {nom }}^{3}$, where $d_{\text {nom }}^{3}=2.54 \mathrm{~mm}$. Similarly, the REV of the $\mathrm{CaCO}_{3}$ packed bed was determined as $24 \times d_{\mathrm{h}}^{3}$ in the study [17], where $d_{\mathrm{h}}^{3}=1.87 \mathrm{~mm}$. The slab is illuminated normally to the surface $A$ by a uniform and collimated flux. Hence, Eqs. (5) and (7) reduce to

$$
\begin{aligned}
& \sigma_{\mathrm{s}, \text { int }, i i}=\frac{\int_{A_{\text {int }}: \hat{\mathbf{s}} \cdot \hat{\mathbf{n}}_{i}>0} \rho_{i j}^{\prime \cap}(\hat{\mathbf{s}}) \exp \left[-\left(\kappa_{\mathrm{d}, i}+\sigma_{\mathrm{s}, \mathrm{d}, i}\right) S^{*}\right] \hat{\mathbf{s}} \cdot \hat{\mathbf{n}}_{i} \mathrm{~d} A_{\text {int }}}{\int_{V_{i}} \exp \left[-\left(\kappa_{\mathrm{d}, i}+\sigma_{\mathrm{s}, \mathrm{d}, i}\right) S^{*}\right] \mathrm{d} V}
\end{aligned}
$$

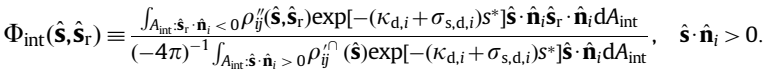

Bouguer's law generally applies locally, in each individual phase, provided the RTE is valid [13]. Analogous relations apply to Eqs. (6) and (8). The MC ray-tracing method is employed to compute the integrals in Eqs. (9) and (10). For an isotropic and continuum-scale homogeneous two-phase medium Eqs. (9) and (10) are independent of the orientation and location of the slab, i.e. continuum-scale radiative properties obtained from Eqs. (9) and (10) are the same as those obtained by averaging the radiative properties for a large number of arbitrarily positioned and oriented slabs. Thus, the averaging volume $V=A s$ can be replaced by a statistically equivalent volume consisting of large number of sub-volumes $V_{k}=A_{k} s$ that are randomly oriented and distributed in $V$. Starting points $\mathbf{r}_{k}$ and directions $\hat{\mathbf{s}}_{k}$ of a large number of stochastic rays are uniformly distributed in either phase and over the solid angle $4 \pi$, respectively. One realization is that $\mathbf{r}_{k}$ and $\hat{\mathbf{s}}_{k}$ are associated with one subvolume $V$. A ray is traced until it either hits the interface between the phases 1 and 2 at distance $s_{k}=\left|\mathbf{r}_{\text {int }}-\mathbf{r}_{k}\right|$ or it leaves the sub-volume $V_{k}$ through the boundary at distance $s_{k}=\left|\mathbf{r}_{\mathrm{w}}-\mathbf{r}_{k}\right|$. Eqs. (9) and (10) are approximated by

$\sigma_{\mathrm{s}, \mathrm{int}, i i} \approx \frac{-\left(\kappa_{\mathrm{d}, i}+\sigma_{\mathrm{s}, \mathrm{d}, i}\right) \sum_{k=1}^{N_{c, i}}\left\{\rho_{i j}^{\prime n}\left(\hat{\mathbf{s}}_{k}\right) \exp \left[-\left(\kappa_{\mathrm{d}, i}+\sigma_{\mathrm{s}, \mathrm{d}, i}\right) \mathrm{s}_{k}\right]\right\}}{\sum_{k=1}^{N_{i}}\left\{1-\exp \left[-\left(\kappa_{\mathrm{d}, i}+\sigma_{\mathrm{s}, \mathrm{d}, i}\right) s_{k}\right]\right\}}$

$\Phi_{\text {int }, i i}\left(\hat{\mathbf{s}}, \hat{\mathbf{s}}_{\mathrm{r}}\right) \equiv \frac{\sum_{k=1}^{N_{\mathrm{c}, i}}\left\{\rho_{i j}^{\prime \prime}\left(\hat{\mathbf{s}}_{k}, \hat{\mathbf{s}}_{\mathrm{r}}\right) \exp \left[-\left(\kappa_{\mathrm{d}, i}+\sigma_{\mathrm{s}, \mathrm{d}, i}\right) s_{k}\right] \hat{\mathbf{s}}_{\mathrm{r}} \cdot \hat{\mathbf{n}}_{i}\right\}}{(4 \pi)^{-1} \sum_{k=1}^{N_{\mathrm{c}, i}}\left\{\rho_{i j}^{\prime \cap}(\hat{\mathbf{s}}) \exp \left[-\left(\kappa_{\mathrm{d}, i}+\sigma_{\mathrm{s}, \mathrm{d}, i}\right) S_{k}\right]\right\}}$

For a non-participaring phase $i$, Eqs. (9) and (10) reduce to

$\sigma_{\mathrm{s}, \text { int }, i i}=\frac{\int_{A_{\text {int }}: \hat{\mathbf{s}} \cdot \hat{\mathbf{n}}_{i}>0} \rho_{i j}^{\prime \cap}(\hat{\mathbf{s}}) \hat{\mathbf{s}} \cdot \hat{\mathbf{n}}_{i} \mathrm{~d} A_{\text {int }}}{V_{i}}$

$\Phi_{\text {int }, i i}\left(\hat{\mathbf{s}}, \hat{\mathbf{s}}_{\mathrm{r}}\right)=\frac{\int_{A_{\text {int }} \hat{\mathbf{s}} \cdot \hat{\mathbf{n}}_{i}<0} \rho_{i j}^{\prime \prime}\left(\hat{\mathbf{s}}, \hat{\mathbf{s}}_{\mathrm{r}}\right) \hat{\mathbf{s}} \cdot \hat{\mathbf{n}}_{i} \hat{\mathbf{s}}_{\mathrm{r}} \cdot \hat{\mathbf{n}}_{i} \mathrm{~d} A_{\text {int }}}{(-4 \pi)^{-1} \int_{A_{\text {int }} \hat{\mathbf{s}} \cdot \hat{\mathbf{n}}_{i}>0} \rho_{i j}^{\prime \cap}(\hat{\mathbf{s}}) \hat{\mathbf{s}} \cdot \hat{\mathbf{n}}_{i} \mathrm{~d} A_{\text {int }}}, \quad \hat{\mathbf{s}} \cdot \hat{\mathbf{n}}_{i}>0$

\subsection{Computed tomography-based geometry}

Computed tomography (CT) is employed to obtain the exact geometry of the selected two-phase media. Tomography data sets consist of $l \times m \times n$ matrices of 8 or 16 bit gray level information, $\Psi_{i, j, k}$. Trilinear interpolation is used to create a continuous gray level function $\Psi(\mathbf{r})$ [16]. At the interface between the phases $\Psi(\mathbf{r})=\Psi_{0}$. Eqs. (11)-(12) are evaluated by intersecting rays with the interface:

$\Psi\left(\mathbf{r}_{k}+s_{k} \hat{\mathbf{s}}_{k}\right)=\Psi_{0}$

The normal vector at the intersection is calculated as

$\hat{\mathbf{n}}_{i}=\frac{\left.\nabla \Psi\right|_{\mathbf{r}_{k}+s_{k} \hat{\mathbf{s}}_{k}}}{|\nabla \Psi|_{\mathbf{r}_{k}+s_{k} \hat{\mathbf{s}}_{k} \mid}}$ 


\section{Results}

\subsection{Continuum-scale radiative properties}

The continuum-scale scattering coefficients $\sigma_{\mathrm{s}, \text { int }, i i}$ and $\sigma_{\mathrm{s}, \mathrm{int}, i j}$, and the scattering phase functions $\Phi_{\text {int }, i i}$ and $\Phi_{\text {int }, i j}$ are determined for the two selected model two-phase media. The computed tomography techniques and the physical parameters of the model media are described in Ref. [16] (RPCs) and in Ref. [17] $\left(\mathrm{CaCO}_{3}\right)$. Smooth surfaces, i.e., specular reflection and the Snell-type refraction, are assumed. The optically thin limit of Snell's law and the Fresnel equation are used.

\subsubsection{SiC RPC}

A $512 \times 512 \times 512$ voxel, equivalent to $5 d_{\mathrm{h}} \times 5 d_{\mathrm{h}} \times 5$ $d_{\mathrm{h}}$, tomographic scan of a 10 ppi SiC RPC foam (Fig. 1a) with calculated porosity $\varepsilon_{\mathrm{p}}=0.86$ and specific surface $A_{0}=1.06 \times 10^{3} \mathrm{~m}^{-1}$ is used. No dependence of the continuum-scale radiative properties $\sigma_{\mathrm{s}, \text { int }, i i}, \sigma_{\mathrm{s}, \text { int }, i j}$, and, $\Phi_{\text {int }, i i}$, and $\Phi_{\text {int }, i j}$ on the discrete-scale absorption and scattering coefficients is found. In Fig. 2, the continuumscale scattering coefficients are shown as a function of the index of refraction ratio $n_{2} / n_{1}$. Because of the total internal reflection (Fresnel interface) the amount of radiation scattered from one phase into the other decreases and the amount of radiation scattered within one phase increase as the ratio of the refractive indices increases. The sum of $\sigma_{\mathrm{s}, \text { int }, i i}$ and $\sigma_{\mathrm{s}, \text { int }, i j}$ remains constant. The scattering phase functions are plotted as a function of the directional cosine $\mu_{\mathrm{s}}=\cos \theta$ in Fig. 3. The phase functions for scattering between phases (1-2, 2-1) are identical. They exhibit a peak in the forward direction, which becomes more pronounced with an increasing refractive index ratio a

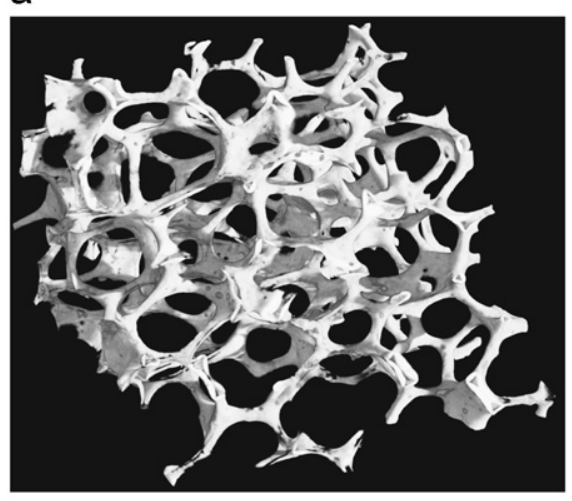

b

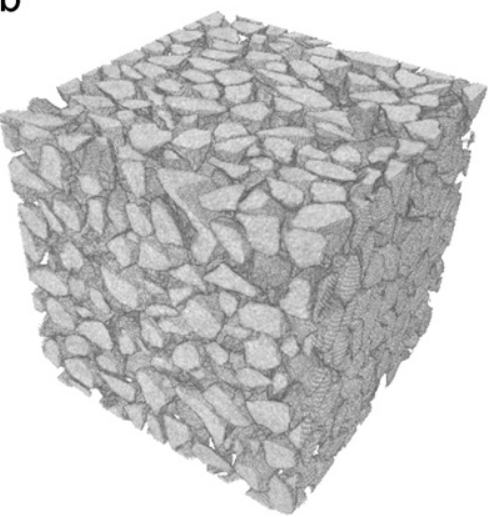

Fig. 1. 3D rendering of the discrete-scale geometry for the (a) RPC and (b) packed bed of $\mathrm{CaCO}_{3}$ particles.

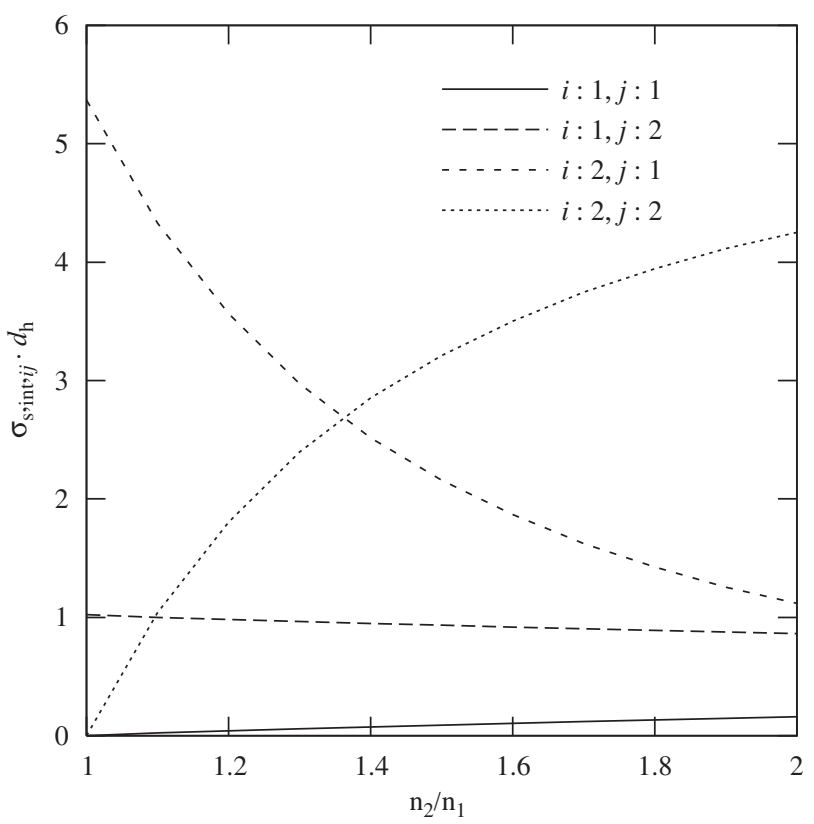

Fig. 2. RPC continuum-scale scattering coefficients as a function of refractive index ration $n_{2} / n_{1}$ (phase 1 : void phase, phase 2 : solid phase). 
$n_{2} / n_{1}$. For decreasing $\mu_{\mathrm{s}}$ the scattering phase functions rapidly decrease to zero. The phase functions for scattering within the phase with the lower refractive index behave similar to scattering associated with transmission between the phases except they do not drop to 0 for backward directions. The phase function for scattering within the phase with the higher refractive index is relatively flat in the forward direction and rapidly approaches zero as a result of the total internal reflection.

\subsection{2. $\mathrm{CaCO}_{3}$ packed bed}

A $512 \times 512 \times 512$ voxel, equivalent to $12 d_{\mathrm{h}} \times 12 d_{\mathrm{h}} \times 12$ $d_{\mathrm{h}}$, tomographic scan of a packed bed of $\mathrm{CaCO}_{3}$ particles (Fig. 1b) with calculated $\varepsilon_{\mathrm{p}}=0.4$ and $A_{0}=1.31 \times 10^{3} \mathrm{~m}^{-1}$ is employed. No dependence of the continuum-scale properties $\sigma_{\mathrm{s}, \text { int }, i i}, \sigma_{\mathrm{s}, \mathrm{int}, i j}, \Phi_{\mathrm{int}, i i}$, and $\Phi_{\mathrm{int}, i j}$ on the discrete-scale absorption and scattering coefficients is found. The continuum-scale scattering coefficients are shown in Fig. 4 as a function of the refractive index ratio $n_{2} / n_{1}$. The scattering phase functions

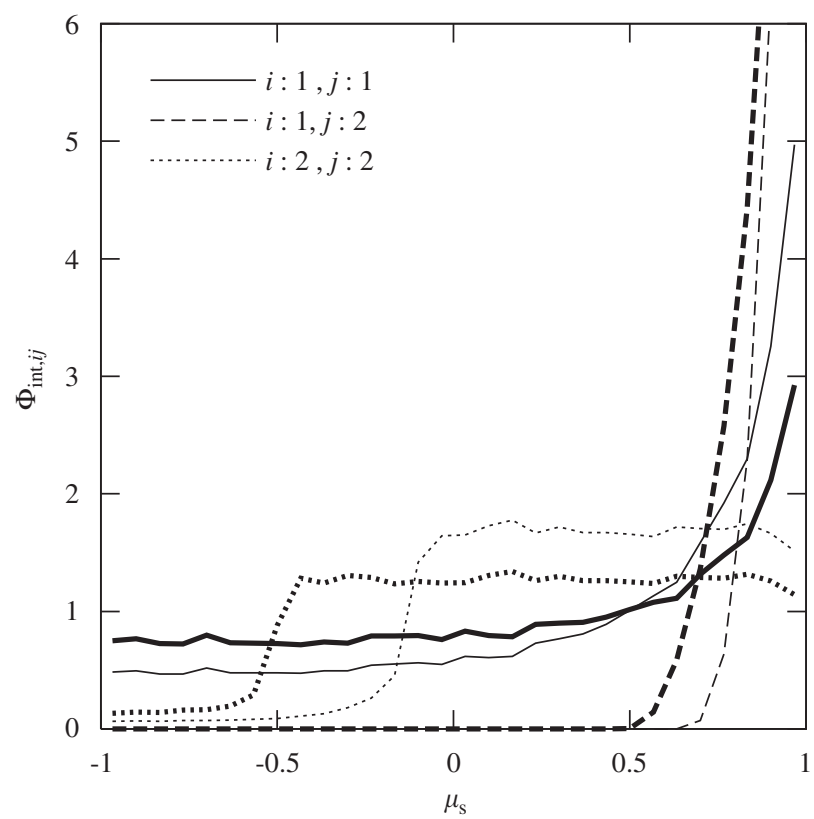

Fig. 3. RPC continuum-scale scattering phase function for scattering between different phases and within phases (phase 1: void phase, phase 2: solid phase) for $n_{1} / n_{2}=1.5$ (thin lines) and $n_{1} / n_{2}=2$ (thick lines).

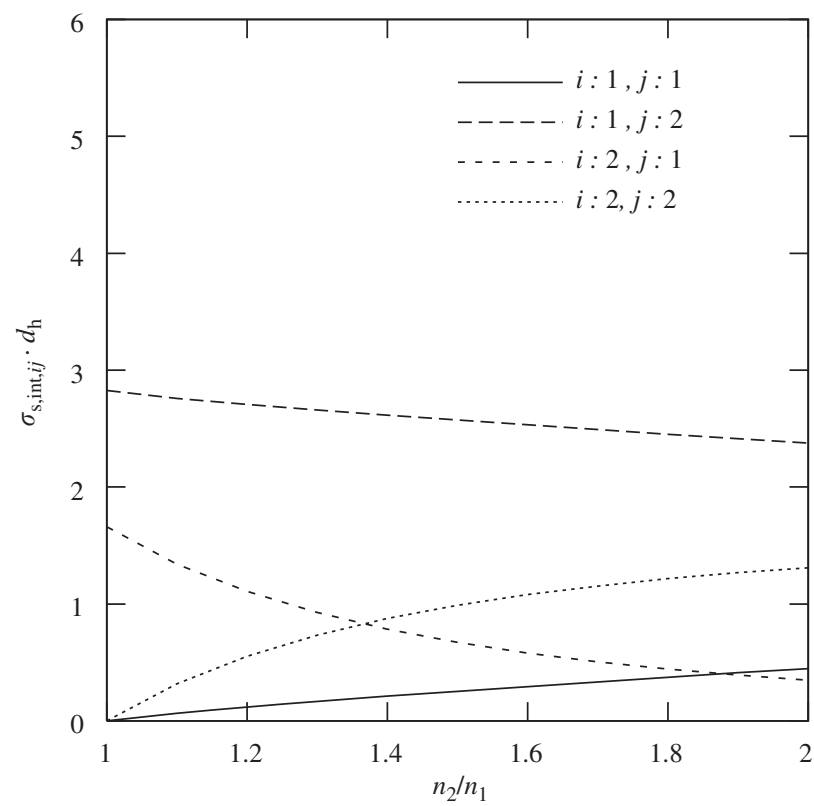

Fig. 4. Packed bed continuum-scale scattering coefficients as a function of refractive index ration $n_{2} / n_{1}$ (phase 1 : void phase, phase 2 : fluid phase). 
behave very similar to the RPC case and are shown in Fig. 5. Since the morphologies of the two samples differ significantly, we conclude that, for the range of porosities and refractive index ratios investigated, the influence of the porous micro-geometry on continuum-scale geometrical optics radiative transfer is minor. However, no systematic investigation of this phenomenon has been undertaken in the present study.

\subsection{Radiative fluxes}

The computational domain for determining discreteand continuum-scale radiative fluxes is depicted in Fig. 6. Discrete-scale and continuum-scale MC simulations are performed for negligible discrete-scale extinction coefficient of one phase and for the refractive index ratio $n_{2} / n_{1}=1.5$. The $x y$-face of a cubic sample is exposed to a uniform collimated beam parallel to the $z$-axis. All faces are exposed to black surroundings at $0 \mathrm{~K}$.

\subsubsection{SiC RPC}

Consider the normalized radiative flux, per phase, averaged over the total medium cross-sectional area, in positive and negative axis directions (denoted by+ and - , respectively). In Fig. 7 , the radiative fluxes along the direction of incidence are plotted. The forward flux in the void phase decreases as radiation passes through the sample. This is partly due to scattering into the solid phase and partly due to radiation losses to the surroundings. The comparably strong underprediction of $\overline{\dot{q}_{z 1}^{+}}$and the coinciding overprediction of $\overline{\dot{q}_{z 2}^{+}}$for continuum-scale simulations is due to local anisotropy in the interface orientation for $z / d_{\mathrm{h}}$ between 1 and 2 , which leads to the transfer of high amounts of radiation from phase 2 to phase 1 . The solid phase forward fluxes first increase due to in-scattering from the void phase and then decrease due to losses to the surroundings.

In Fig. 8, the radiative fluxes perpendicular to the direction of incidence are shown. Due to the axial symmetry of the problem, only $x$-axis fluxes are given, while $y$-axis fluxes are omitted. Fluxes are zero at the black boundary for the inward-facing directions. They then increase due to in- and forward-scattering, reach a maximum and decrease again due to out- and backscattering. Direct simulations (thin lines) and continuum simulations (thick lines) show good agreement: RMS deviation between the results of the two approaches are in the range between $0.71 \%$ of $\dot{q}_{0}$ (phase $1, \dot{q}_{x}^{-}$) and $3.91 \%$ of $\dot{q}_{0}$ (phase $2, \dot{q}_{x}^{+}$). Fluctuations in the fluxes obtained by

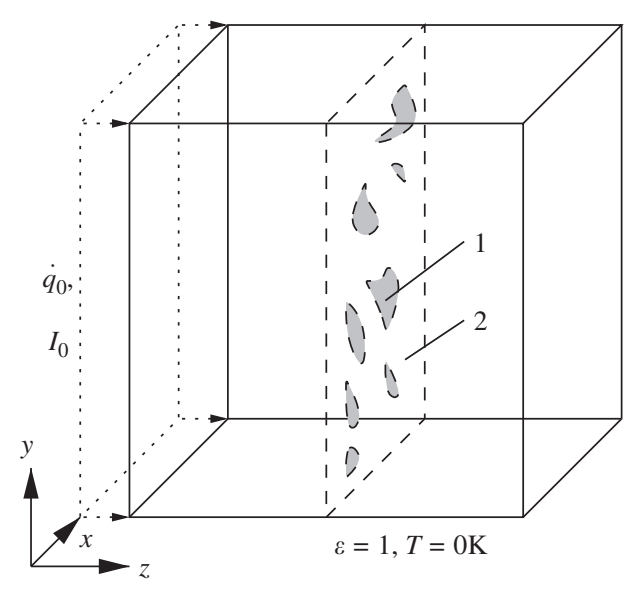

Fig. 6. Schematic diagram of the computational domain for determining the discrete and continuum-scale radiative fluxes.

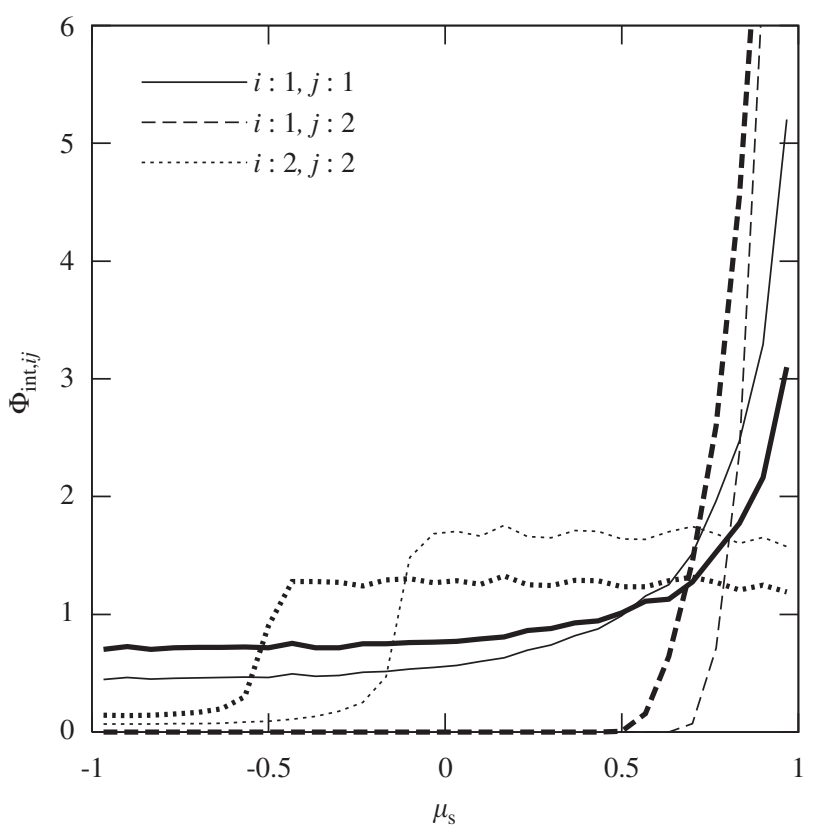

Fig. 5. Packed bed continuum-scale scattering phase function for scattering between different phases (phase 1: void phase, phase 2: fluid phase) for $n_{1} / n_{2}=1.5$ (thin lines) and $n_{1} / n_{2}=2$ (thick lines). 


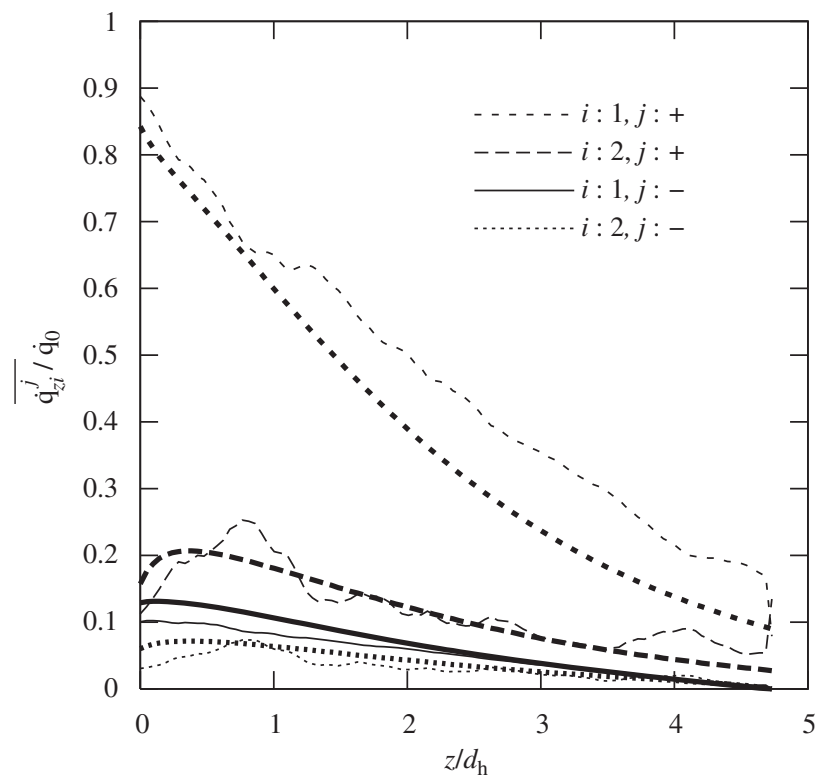

Fig. 7. Mean radiative fluxes for the RPC along the $z$-direction and into the forward (+) and backward (-) hemisphere using discrete (thin lines) and continuum-scale (thick lines) simulations.

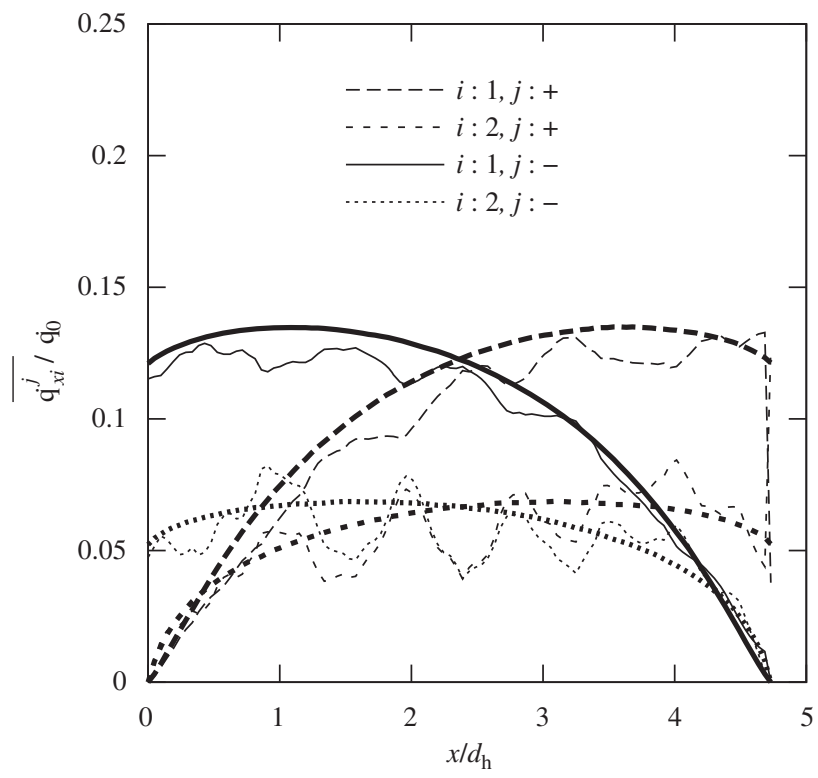

Fig. 8. Mean radiative fluxes for the RPC along the $x$-direction and into the forward $(+)$ and backward ( - ) hemisphere using discrete- (thin lines) and continuum-scale (thick lines).

the direct simulation are due to statistical variation in the local geometry, and due to the finite sample size.

The direct numerical simulation based on $4 \times 10^{6}$ rays for $n_{1} / n_{2}=1 / 1.9$ was completed within $2514 \mathrm{~s}$. In comparison, the determination of the continuum properties for the same situation was completed within $197 \mathrm{~s}$, and the continuum simulation was completed within $162 \mathrm{~s}$. Computational time is hence reduced to $14 \%$ of the time needed by direct simulation. If the determination of properties, which only needs to be carried out once, is neglected, computational time is further reduced to $6.4 \%$.

\subsection{2. $\mathrm{CaCO}_{3}$ packed bed}

Consider the normalized radiative fluxes, per phase, averaged over the medium cross-sectional area, in positive and negative axis directions (denoted + and respectively). In Fig. 9, the radiative fluxes along the direction of incidence are plotted. Their variation follows that obtained for the RPC. The forward flux in the void 
phase decreases as radiation passes through the sample. The forward fluxes in the solid phase exhibit a maximum at the distance of approximately $0.5 d_{\mathrm{h}}$ from the irradiated boundary. The radiative fluxes perpendicular to the direction of incidence are shown in Fig. 10. Only the fluxes along the $x$-direction are reported. Their variation follows, again, that obtained for the RPC. Direct simulations (thin lines) and continuum simulations show good agreement: RMS deviation between results of the two approaches are in the range between $0.25 \%$ of $\dot{q}_{0}$ (phase $1, \dot{q}_{x}^{-}$) and 1.32 of $\dot{q}_{0}$ (phase $1, \overline{\dot{q}}_{z}^{+}$). Fluctuations in the fluxes obtained in the direct simulation result from the statistical variations of the local geometry, and from the finite sample size.

\subsection{MC accuracy considerations}

Convergence of the MC algorithms used for the determination of the discrete-scale and the continuum-scale radiative fluxes is examined for the 2-norm of the normalized

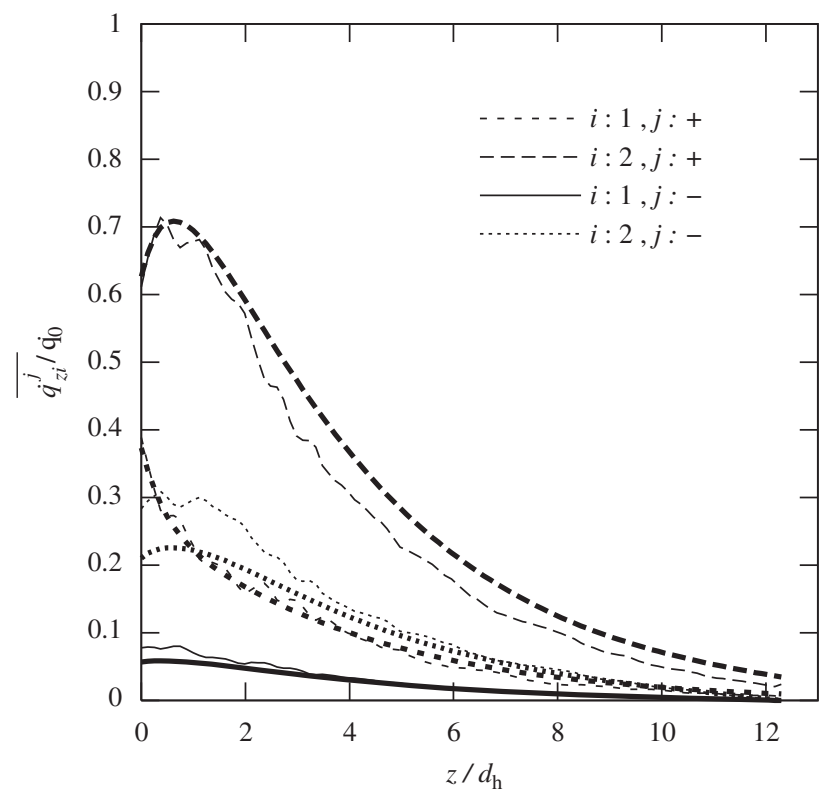

Fig. 9. Mean radiative fluxes for the $\mathrm{CaCO}_{3}$ packed bed along the $z$-direction and into the forward $(+)$ and backward $(-)$ hemisphere using discrete- (thin lines) and continuum-scale (thick lines) simulations.

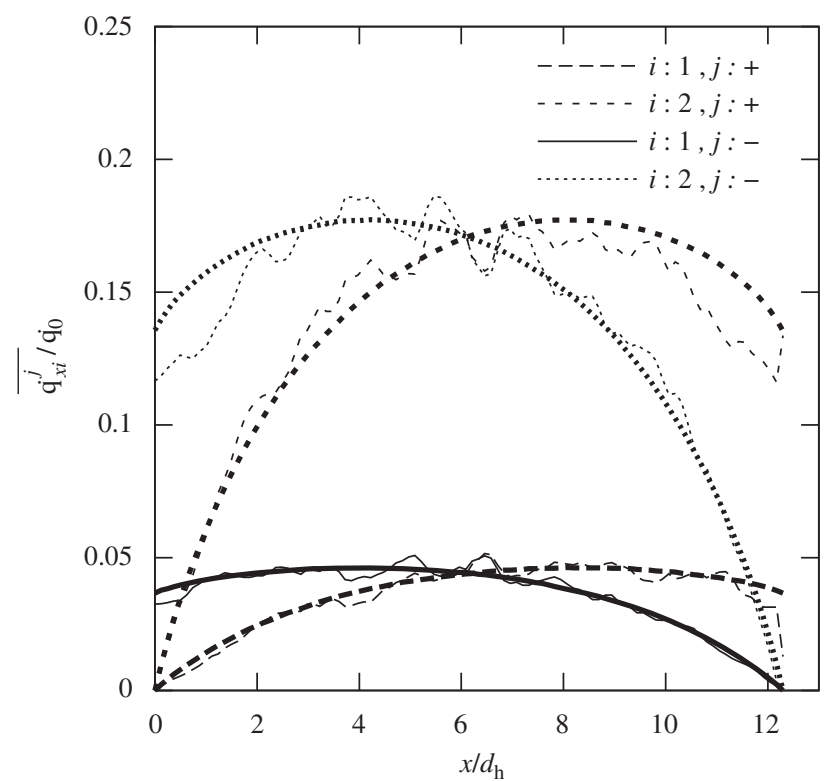

Fig. 10. Mean radiative fluxes for the $\mathrm{CaCO}_{3}$ packed bed along the $x$-direction and into the forward $(+)$ and backward $(-)$ hemisphere using discrete(thin lines) and continuum-scale (thick lines) simulations. 
a

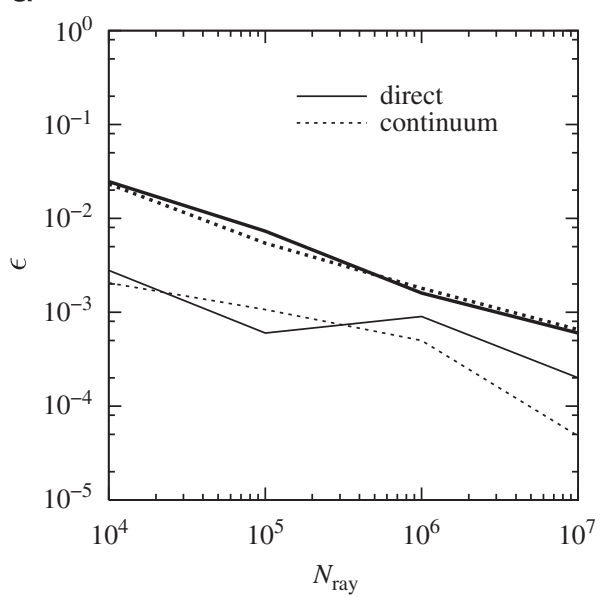

b

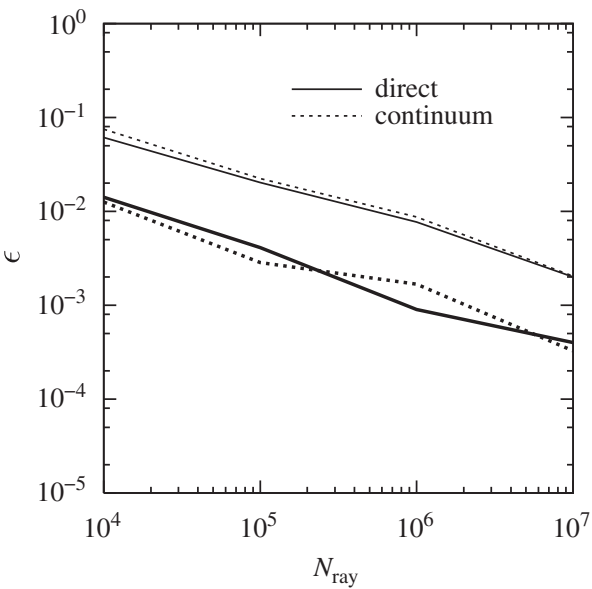

Fig. 11. Normalized 2-norm of the radiative fluxes averaged over the $x y$-face for (a) the solid phase and (b) the fluid phase in the forward direction. The discrete-scale and continuum-scale MC results are given for SiC RPC (thin lines) and packed bed of $\mathrm{CaCO}_{3}$ (thick lines).

radiative flux, per phase, averaged over the total medium cross-sectional area, in positive or negative axis directions for $10^{4}, 10^{5}, 10^{6}$, and $10^{7}$ stochastic rays, normalized with respect to the reference solution for $10^{8}$ rays, $\epsilon=\left\|\mathbf{y}-\mathbf{y}_{\text {ref }}\right\|_{2} /$ $\left\|\mathbf{y}_{\text {ref }}\right\|_{2}$, with $\mathbf{y}$ being the computed vector of the corresponding normalized radiative flux for the corresponding number of rays. The results are shown for two selected radiative fluxes in Fig. 11. As expected for the MC methods, the convergence order of $\epsilon$ is approximately 0.5, i.e. $\epsilon$ decreases by 0.5 decades per decade increase in $N_{\text {ray. }}$.

\section{Conclusions}

The mathematical model of continuum radiative transfer in two-phase media with the phases in the limit of geometrical optics derived previously has been numerically validated in the present study. Continuum-scale radiative properties of reticulate porous ceramics and packed beds of calcium carbonate were determined. The radiative fluxes obtained by the continuum-scale approach were compared with those obtained by the discrete-scale approach. The continuum-scale radiative properties were determined by employing computed tomography-based Monte Carlo techniques. These properties primarily depend on the discrete-scale radiative and optical properties, and on the pore/particle/strut characteristic size and volume fraction of each phase.

The radiative fluxes computed using the direct, discretescale simulations, and the continuum-scale simulations are in good agreement. Due to statistical variations of the local geometry and finite sample sizes discrete-scale simulations exhibit strong fluctuations, which are not observed in the continuum-scale simulations. For all practical applications, where the discrete-scale radiative flux and divergence of radiative flux distributions are not needed, the continuumscale modeling approach is advantageous as it provides physically correct, practically relevant information at a significantly lower computational cost.

\section{References}

[1] Lipiński W, Steinfeld A. Heterogeneous thermochemical decomposition under direct high-flux irradiation. International Journal of Heat and Mass Transfer 2004;47:1907-16.

[2] Schunk LO, Lipiński W, Steinfeld A. Ablative heat transfer in a shrinking packed-bed of $\mathrm{ZnO}$ undergoing solar thermal dissociation. AIChE J 2009;48:1510-6.

[3] Jäger K, Lipiński W, Katzgraber HG, Steinfeld A. Determination of thermal radiative properties of packed-bed media containing a mixture of polydispersed particles. International Journal of Thermal Sciences 2009;150:502-8.

[4] Howell JR, Hall MJ, Ellzey JL. Combustion of hydrocarbon fuels within porous inert media. Progress in Energy and Combustion Science 1996;22:121-45.

[5] Barra AJ, Diepvens G, Ellzey JL, Henneke MR. Numerical study of the effects of material properties on flame stabilization in a porous burner. Combustion and Flame 2003;134:369-79.

[6] Barra AJ, Ellzey JL. Heat recirculation and heat transfer in porous burners. Combustion and Flame 2004;137:230-41.

[7] Viskanta R. Radiative Transfer in Combustion Systems: Fundamentals and Applications. Begell House, Inc.; 2005.

[8] Muir JF, Hogan Jr RE, Skocypec RD, Buck R. Solar reforming of methane in a direct absorption catalytic reactor on a parabolic dish: I-test and analysis. Solar Energy 1994;52:467-77.

[9] Skocypec RD, Hogan Jr RE, Muir JF. Solar reforming of methane in a direct absorption catalytic reactor on a parabolic dish: II-modeling and analysis. Solar Energy 1994;52:479-90.

[10] Buck R, Muir JF, Hogan Jr RE. Carbon dioxide reforming of methane in a solar volumetric receiver/reactor: the CAESAR project. Solar Energy Materials 1991;24:449-63.

[11] Keshtkar MM, Gandjalikhan Nassab SA. Nasr MRJ. Heat transfer characteristics of a cylindrical porous radiant air heater under the influence of a two-dimensional axisymmetric radiative field. Proceedings of the Institution of Mechanical Engineers, Part A: Journal of Power and Energy 2009;223:913-23.

[12] Howell JR, Siegel R, Mengüç MP. Thermal Radiation Heat Transfer. 5th edition. CRC Press; 2010.

[13] Modest MF. Radiative Heat Transfer. 2nd ed. Academic Press; 2003

[14] Tancrez M, Taine J. Direct identification of absorption and scattering coefficients and phase function of a porous medium by a Monte Carlo technique. International Journal of Heat and Mass Transfer 2004; 47:373-83.

[15] Zeghondy B, Iacona E, Taine J. Determination of the anisotropic radiative properties of a porous material by radiative distribution function identification (RDFI). International Journal of Heat and Mass Transfer 2006;49:2810-9.

[16] Petrasch J, Wyss P, Steinfeld A. Tomography-based Monte Carlo determination of radiative properties of reticulate porous ceramics. Journal of Quantitative Spectroscopy and Radiative Transfer 2007; 105:180-197. 
[17] Haussener S, Lipiński W, Petrasch J, Wyss P, Steinfeld A. Tomographic characterization of a semitransparent-particle packed bed and determination of its thermal radiative properties. Journal of Heat Transfer 2009;131:072701.

[18] Haussener S, Coray P, Lipiński W, Wyss P, Steinfeld A. Tomographybased heat and mass transfer characterization of reticulate porous ceramics for high-temperature processing. Journal of Heat Transfer 2010;132:023305.

[19] Haussener S, Lipiński W, Wyss P, Steinfeld A. Tomography-based analysis of radiative transfer in reacting packed beds undergoing a solid-gas thermochemical transformation. Journal of Heat Transfer 2010;132:014003.

[20] Lipiński W, Petrasch J, Haussener S. Application of the spatial averaging theorem to radiative heat transfer in two-phase media. Journal of Quantitative Spectroscopy and Radiative Transfer 2010; 111:253-8.

[21] Lipiński W, Keene D, Petrasch J, Haussener S, Petrasch J. Continuum radiative heat transfer modeling in media consisting of optically distinct components in the limit of geometrical optics. Journal of Quantitative Spectroscopy and Radiative Transfer 2010;111: 2474-80.

[22] Consalvi JL, Porterie B, Loraud JC. A formal averaging procedure for radiation heat transfer in particulate media. International Journal of Heat and Mass Transfer 2002;45:2755-68.

[23] Lipiński W, Z'Graggen A, Steinfeld A. Transient radiation heat transfer within a nongray nonisothermal absorbing-emitting-scattering suspension of reacting particles undergoing shrinkage. Numerical Heat Transfer Part B-Fundamentals 2005;47:443-57.

[24] Whitaker S. The Method of Volume Averaging. Kluwer Academic Publishers; 1999.

[25] Kaviany M. Principles of Heat Transfer in Porous Media. Springer; 1995.

[26] Bear J. Dynamics of Fluids in Porous Media. New York: Dover Publications; 1972. 